\title{
Granular anchors for stabilising slopes
}

\author{
V. SIVAKUMAR*, J. LIVELY*, B. SOLAN $\dagger$ and J. BLACK +
}

\begin{abstract}
A series of small-scale tests was undertaken to verify if granular anchors could be used as a slope stabilisation technique. The nature of the material used and the resulting loading configuration are described here. The work confirms that the inclusion of anchors within a slope mass, irrespective of their number or orientation, significantly enhances the capacity and ductility of the failure mode. The small-scale nature of this research did influence the observed capacities, but the overarching hypothesis was confirmed. A simple analysis method is proposed that allows designers to accurately remediate natural or man-made slopes using existing analytical methods for slope stability.
\end{abstract}

KEYWORDS: anchors; gravels; ground improvement; landslides; retaining walls; soil nailing

ICE Publishing: all rights reserved

\section{INTRODUCTION}

Granular columns are traditionally used to improve weak deposits by enhancing the bearing capacity and limiting the settlement of foundations. The effectiveness of this ground improvement technique is essentially controlled by the strength and stiffness of the soil confining the granular columns, particularly at shallow depths where the columns are prone to bulging. Phanikumar \& Ramachandra Rao (2000), Phanikumar et al. (2004), Liu et al. (2006), Srirama Rao et al. (2007), Madhav et al. (2008), Phanikumar et al. (2008) and Sivakumar et al. (2013) looked at extending the applicability of this technique to resisting tension or pullout forces. These modified granular anchors consist of a base plate with a centrally located tendon (cable or metallic rod) encased in compacted granular backfill. The tendon is used to transmit the applied load to the column base via the circular base plate, which compresses the granular material to form the anchor. Under loading conditions, the bulging location of the granular anchor is at depth, where the strength and stiffness of the surrounding soil are significantly higher, thereby augmenting the capacity of the section. Sivakumar et al. (2013) examined the behaviour of granular anchors for a range of lengths $(L)$ and diameters (D). This work concluded that the traditional bulging mechanism is the predominant mode of failure when the $L / D$ ratio is greater than 5 . The authors also carried out parallel studies on concrete anchors of similar $L / D$ ratios and concluded that the capacity of both granular and concrete anchors were similar. However, the deformation responses of the granular anchors were more ductile, as opposed to the brittle failure mode of the concrete anchors.

Granular anchors have been used to prevent uplift caused by flooding (Liu et al., 2006) and to resist heaving of foundations in expansive clays (Srirama Rao et al., 2007). This novel system could have much wider applications in the

Manuscript received 15 June 2014; first decision 24 July 2014; accepted 1 October 2014.

Published online at www.geotechniqueletters.com on 29 October 2014.

*School of Architecture, Planning and Civil Engineering, Queen's University Belfast, Belfast, UK

$\uparrow$ School of the Built Environment, University of Ulster, Newtownabbey, Co. Antrim, UK

Department of Civil and Structural Engineering, University of Sheffield, Sheffield, UK construction industry, for example to enhance the stability of retaining structures, rock faces, natural and man-made slopes, or sheet pile. Granular anchors can also serve as effective drainage systems to prevent the build-up of excessive pore water pressure in natural and man-made slopes. This paper presents some preliminary data obtained from a series of laboratory-based experiments carried out on small-scale model slopes. Two slope angles and two anchor layout and orientations were examined within this study.

\section{MODEL STUDY}

Figure 1 shows the cross-section of the model box in which a slope was constructed using a moist gravelly sand. The key dimensions of the test arrangement are also shown in Fig. 1. The material was mixed with $5 \%$ kaolin to constitute the soil test bed. The gravelly sand had a particle size distribution of $D_{10}=0.1 \mathrm{~mm}, D_{30}=0.25 \mathrm{~mm}$ and $D_{50}=0.7 \mathrm{~mm}\left(D_{60} / D_{10}=7 \cdot 0\right.$ and $\left.D_{30}{ }^{2} / D_{60} D_{30}=0 \cdot 9\right)$. The resulting composite material was mixed at 5\% water content using a large rotating drum mixer. Post-mixing, the material was stored in large sealed plastic bins. In total, ten tests were conducted (see Table 1 for details). Each time, the soil bed material was reused to ensure uniformity of composition within the soil mass. When using the granular anchors, the anchor material was carefully extracted from the failed mass before reusing to limit contamination in further tests. During the tests, the soil bed was covered with cling film to avoid moisture loss. For each test, the box was filled in a series of five layers, each layer being compacted uniformly using a vibrating plate. The final average bulk density of the compacted soil was $1850 \mathrm{~kg} / \mathrm{m}^{3}$. Upon filling of the box, a $45^{\circ}$ slope was formed; this was cut back to leave a $60^{\circ}$ slope if needed, depending on the testing requirements.

A hand auger was used to bore holes $(32 \mathrm{~mm}$ diameter and $500 \mathrm{~mm}$ length, space permitting) in the soil bed through to the back or base of the box, whichever was reached first depending on the orientation of the anchors (Fig. 1). Following this, $500 \mathrm{~mm}$ long steel rods $(3 \mathrm{~mm}$ diameter) connected to a $32 \mathrm{~mm}$ diameter base plate were inserted into the holes. Crushed basalt, with particle sizes of $2 \cdot 36-3.35 \mathrm{~mm}$ and a friction angle of $42^{\circ}$, was fed into the hole in shallow layers $(\approx 100 \mathrm{~mm}$ deep) before being compacted using a special tamping rod. The compaction unit was a hollow shaft with a $30 \mathrm{~mm}$ diameter disc at the 


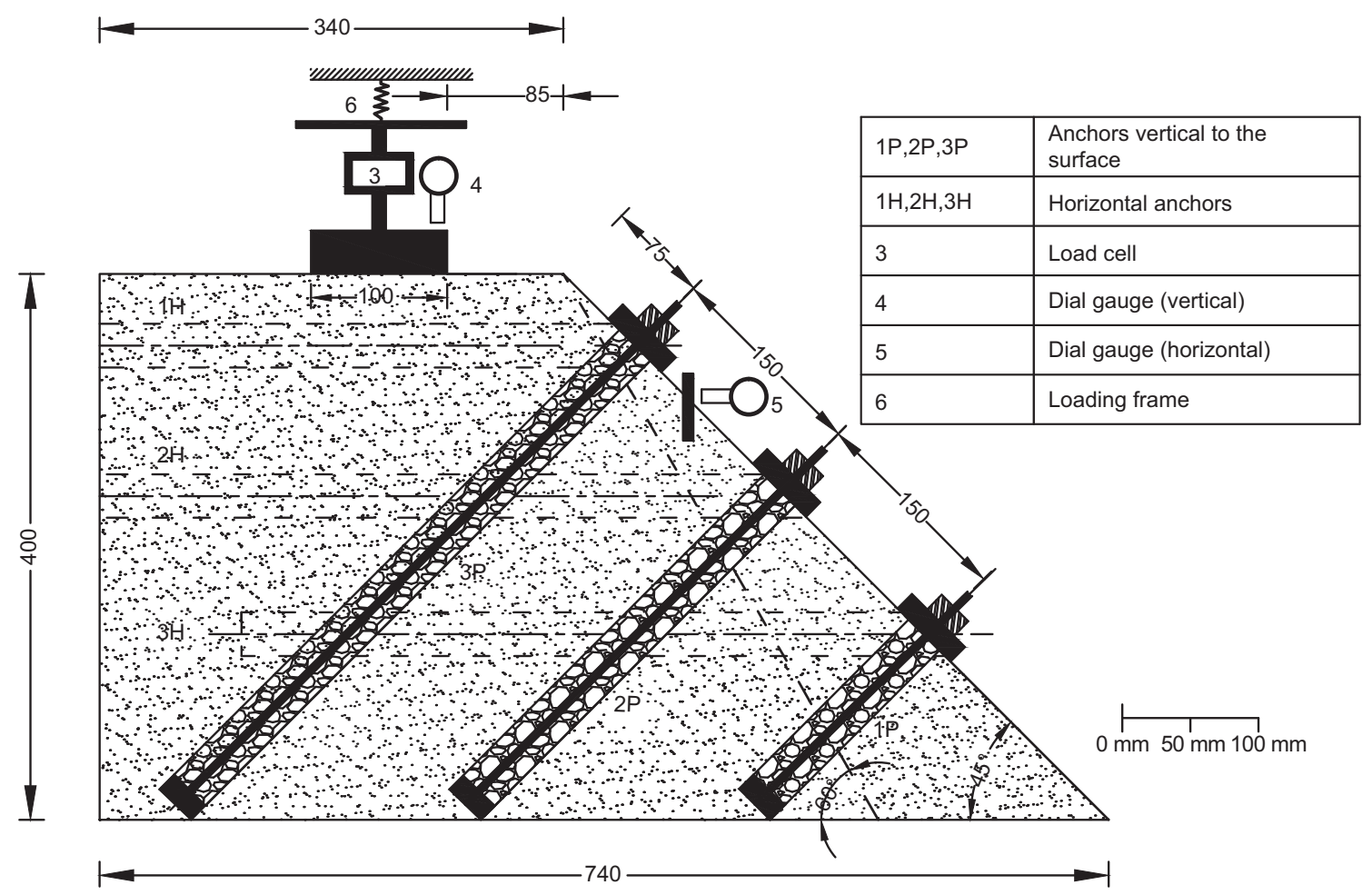

(a) Cross-section

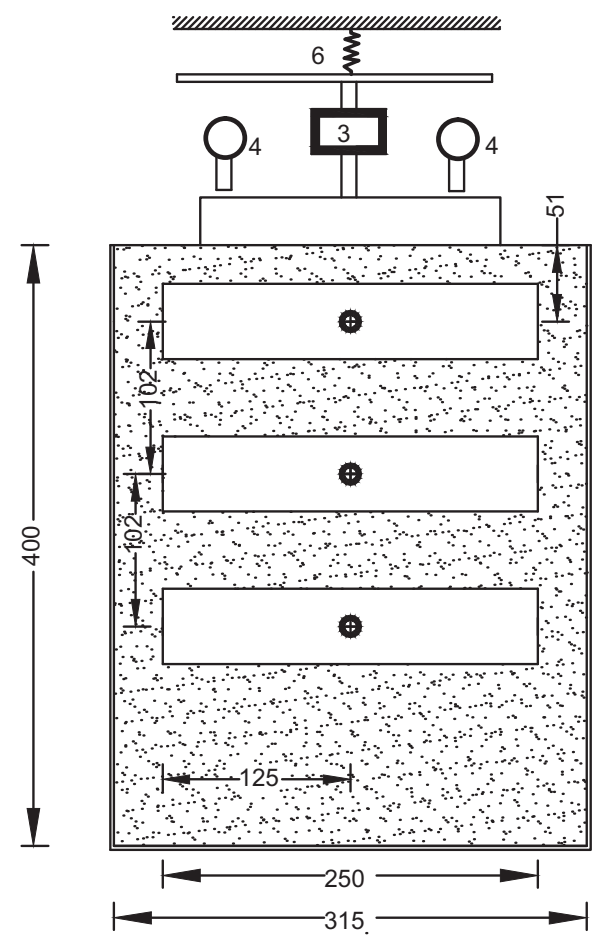

(b) Front view (three anchors)

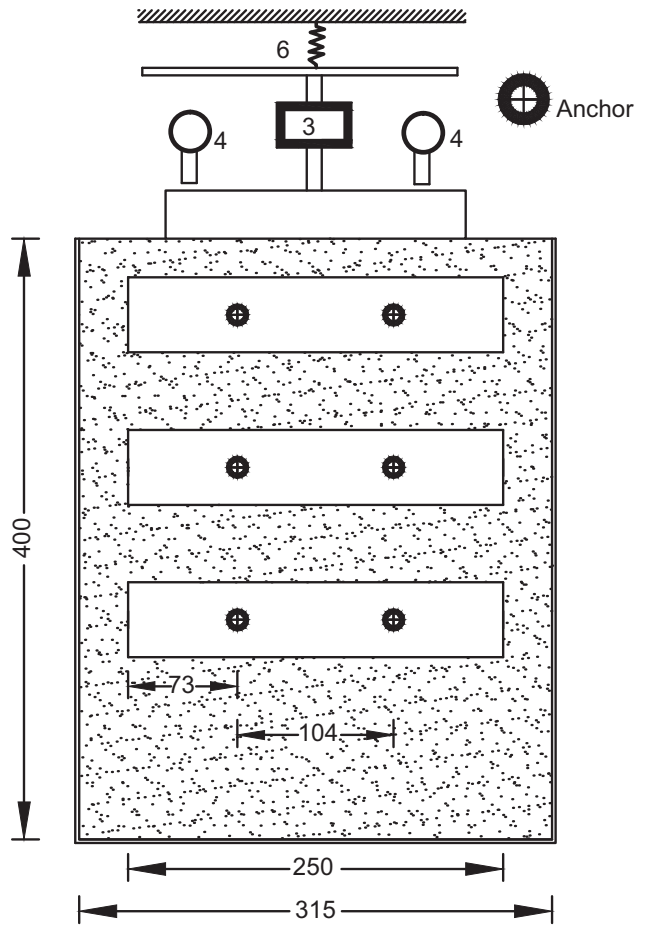

(c) Front view (six anchors)

Fig. 1. Testing arrangement (dimensions in $\mathrm{mm}$ )

end, which could fit around the steel rod and go down the hole. Each layer was compacted 12 times in order to achieve uniform density throughout the length of the anchor and between all anchors respectively. Upon completion of each column, a $250 \mathrm{~mm} \times 40 \mathrm{~mm} \times 5 \mathrm{~mm}$ steel capping plate was bolted on to the protruding steel rods.

Ideally, full-scale testing is needed to confirm the applicability and behaviour of the anchor system to man-made or natural slopes. Before proceeding to the confirmatory studies, possibly using centrifuge, the arrangement shown in Fig. 1 was developed to test the veracity of the hypothesis. Pressure was applied to the top of the test bed by the use of a compression frame as shown in Fig. 1. The required slope failure was induced by the use of a rigid loading plate (100 mm wide, $200 \mathrm{~mm}$ deep) applied to the test arrangement at a rate of $1 \mathrm{~mm} / \mathrm{min}$. This rate is comparable with the rate of test for an unconsolidated undrained triaxial test. Naturally, the small-scale nature of the arrangement was of 
Table 1. Details of experiments

\begin{tabular}{|c|c|c|c|c|c|c|c|c|c|c|}
\hline & \multicolumn{10}{|c|}{ Test } \\
\hline & 1 & 2 & 3 & 4 & 5 & 6 & 7 & 8 & 9 & $10^{\mathrm{b}}$ \\
\hline $\begin{array}{l}\text { Slope angle } \\
\text { No. of anchors } \\
\text { Anchor orientation }^{\text {a }} \\
\text { Anchor arrangement }\end{array}$ & $\begin{array}{c}45^{\circ} \\
0 \\
- \\
-\end{array}$ & $\begin{array}{c}60^{\circ} \\
0 \\
- \\
-\end{array}$ & $\begin{array}{c}45^{\circ} \\
3 \\
\mathrm{H} \\
1 \times 3\end{array}$ & $\begin{array}{c}60^{\circ} \\
3 \\
P \\
1 \times 3\end{array}$ & $\begin{array}{c}60^{\circ} \\
3 \\
\mathrm{H} \\
1 \times 3\end{array}$ & $\begin{array}{c}45^{\circ} \\
6 \\
P \\
2 \times 3\end{array}$ & $\begin{array}{c}45^{\circ} \\
3 \\
P \\
1 \times 3\end{array}$ & $\begin{array}{c}60^{\circ} \\
6 \\
\mathrm{P} \\
2 \times 3\end{array}$ & $\begin{array}{c}60^{\circ} \\
2 \\
\mathrm{H} \\
1 \times 2\end{array}$ & $\begin{array}{r}45^{\circ} \\
0 \\
- \\
-\end{array}$ \\
\hline
\end{tabular}

${ }^{\mathrm{a}} \mathrm{H}$, horizontal; $\mathrm{P}$, perpendicular

${ }^{b}$ Note: test 10 is a repeat of test 1 to ensure experimental quality control and repeatability of results

concern at the outset of the experimental programme. A review of the failure surfaces of test 1 and test 10 (see Table 1) confirmed that the failure mode of the unreinforced section was almost tangential to the bottom of the mould as expected and was extensively two-dimensional in nature. The primary failure surface was located under the centre of the hydraulic loading ram. This confirmed that the side of the model shown in Figs 1(b) and (c) had limited influence on the shape of the failure surface (Whitlow, 1995). The authors accept the simplicity of the testing arrangement, but are confident that the results will yield qualitative results to support their proposed future centrifuge and full-scale testing application. The experimental quality control and the consistent nature of the soil bed reuse are confirmed by the results shown in Fig. 2. A comparison of the performance of unreinforced $45^{\circ}$ and $60^{\circ}$ slopes is also shown in Fig. 2. As per the repeat tests, loaddeformation curves were produced under identical loading and model side slope configuration $\left(45^{\circ}\right)$, one at the start of the programme and one at the end. The general deformation behaviour of the slope was similar in the two tests. The maximum difference between the peak recorded resistances was approximately $0 \cdot 3 \mathrm{kN}$. The displacement differences were very small when the slopes eventually reached their residual failure conditions with visible evidence of slope failure.

\section{RESULTS AND DISCUSSION}

In an effort to mitigate the small-scale nature of the study, the decision was made to use a normalised presentation to compare the results of the plain and reinforced sections. The normalised results were created by dividing the unreinforced peak load (denoted $L_{\mathrm{o}}$ in the relevant figures)

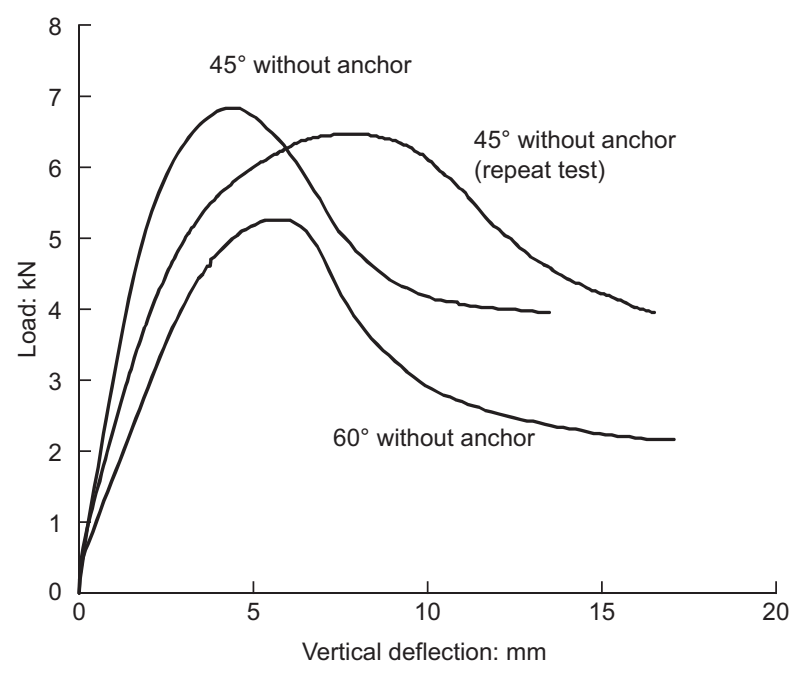

Fig. 2. Unanchored slopes and associated deflection at the time of peak load (denoted as $V_{\mathrm{o}}$ and $H_{\mathrm{o}}$, respectively, for vertical deflection and horizontal deformation in the relevant figures) against the observed reinforced results. The peak loading capacity corresponds to the failure load within the soil mass, beyond which the integrity of the slope is compromised. Figure 3 compares the vertical load-displaced profile and the associated horizontal movements of the plain slope with those of slopes reinforced with three granular anchors, located either in the horizontal direction or perpendicular to the slope face. The performance was clearly enhanced by the inclusion of granular anchors. A comparison of the unreinforced peak strength demonstrated that a similar configuration with three perpendicular anchors produced an increase in capacity of approximately 60\%. A similar comparison but using horizontal anchors produced a $75 \%$ increase in capacity. In both anchored cases, there was no evidence of residual stress within the soil mass (failure condition) and the surcharge loading continued to increase at a slow rate even at termination of the test. The slope with anchors positioned in the horizontal direction performed better than the other configuration. The reason for this will be discussed later in this note. Figure 3(b) demonstrates that the inclusion of anchors reduced the slope deformation. At a normalised vertical displacement $V / V_{\mathrm{o}}=1$ (corresponding to the peak state of the unreinforced slope), the corresponding normalised lateral deformations $\left(H / H_{\mathrm{o}}\right)$ are 0.55 and 0.40 depending on the anchor orientation. This equates to a reduction in side slope movement of about $50-60 \%$.

Figure 4 illustrates the influence of increasing the number of horizontal anchors from three to six on the performance of the same slope profile. The benefits of additional anchors are significant only at large displacement and the relevant performance at the early stage of loading is generally unaffected. However, increasing the number of anchors from three to six has clearly reduced the lateral movement of the slope due to the increased modules of rigidity of the composite mass.

The investigation was extended to examine the effectiveness of granular anchors for stabilising steeper slopes (i.e. slope angle at $60^{\circ}$ to horizontal). Figure 5 shows the loaddisplacement profile of the steeper slope, reinforced with three horizontal or perpendicular anchors. In the case of steepened slopes, when compared with the plain section, the inclusion of anchors in the slope resulted in a capacity increase of 60 and $110 \%$ for the perpendicular and horizontal anchors respectively. In both cases, the anchors effectively prevented the slope producing a residual state failure. The horizontal movement of the slopes was also reduced by their use (Fig. 5(b)). Comparing Figs 3(b) and 5(b) at $V / V_{\mathrm{o}}=1$ indicates similar horizontal displacement profiles, with the $60^{\circ}$ reinforced slope showing slightly lower deformations than the $45^{\circ}$ slope. This may be due to the altered modules of rigidity of the overall soil mass. 


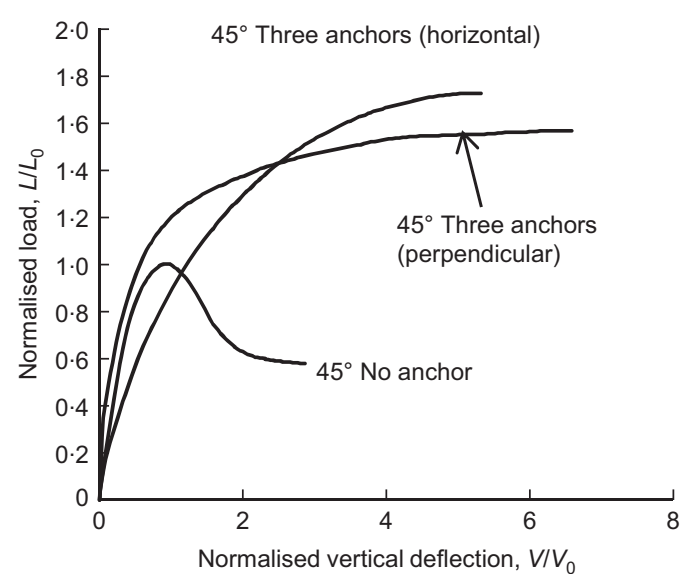

(a)

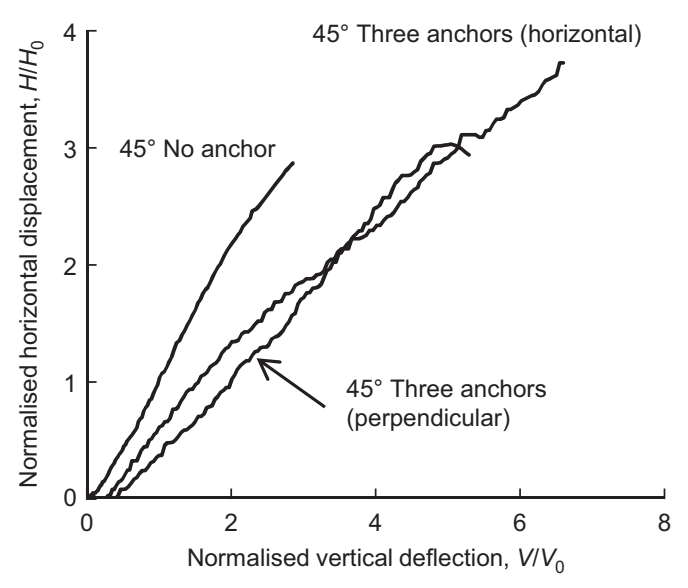

(b)

Fig. 3. Performance of slopes reinforced with three horizontal and perpendicular anchors ( $45^{\circ}$ slopes): (a) load versus vertical displacement; (b) horizontal and vertical displacement

Figure 6 demonstrates the influence of anchor numbers on the behaviour of the steeper slope. All anchors were installed in the perpendicular direction. When compared with the plain slope, it is evident that the addition of extra anchors results in increases in load-carrying capacity of nearly $60 \%$ for three anchors and $175 \%$ for six anchors. Furthermore, the additional anchors added improved ductility to the section.

To assess the potential influence of the reinforcing within the anchors on the test results, particularly in the horizontal direction, one test was conducted with only two anchors (anchors $1 \mathrm{H}$ and 2H, Fig. 1). Figure 7 demonstrates that the slope with two anchors had approximately $60 \%$ more capacity than the plain slope. However, there is evidence of a reduction in capacity when compared with the three-anchor arrangement. Interestingly, the slope with only two anchors had a relatively ductile deformation response (approximate elasto-plastic deformation response) when compared with the unreinforced section. The absence of anchor $1 \mathrm{H}$ (located under the loading plate) did reduce the overall performance and this suggests that the enhanced performance in the case of three granular anchors may be partly attributable to the interaction between the loading plate and the steel bar in anchor $3 \mathrm{H}$. In the case of the three-anchor model, the test was terminated early due to a lack of travel in the compression frame, thus the peak value shown in the two-anchor model had not been reached. Therefore, the final load and associated deflection shown for the three-anchor case are lower bound values of the actual failure load.

The results of this small-scale study have verified the hypothesis that granular anchors can be used to stabilise slopes. Anchors installed in the horizontal direction produced better performance than those installed perpendicular to the slope face. Increasing the number of anchors was shown to augment capacity and ductility at failure, but the enhanced performance must be judged in relation to the cost of installing anchors at full scale. The authors concede that the small-scale nature of the tests may have influenced the results, with some of the enhanced performance being attributable to the steel rod running through the anchors, particularly for anchors close to the loading plate. However, in practice (i.e. at full scale), such interference would not take place. In terms of full-scale applications, installing horizontal anchors is not practical despite offering enhanced drainage within the soil mass. The use of perpendicular anchors will also allow effective drainage

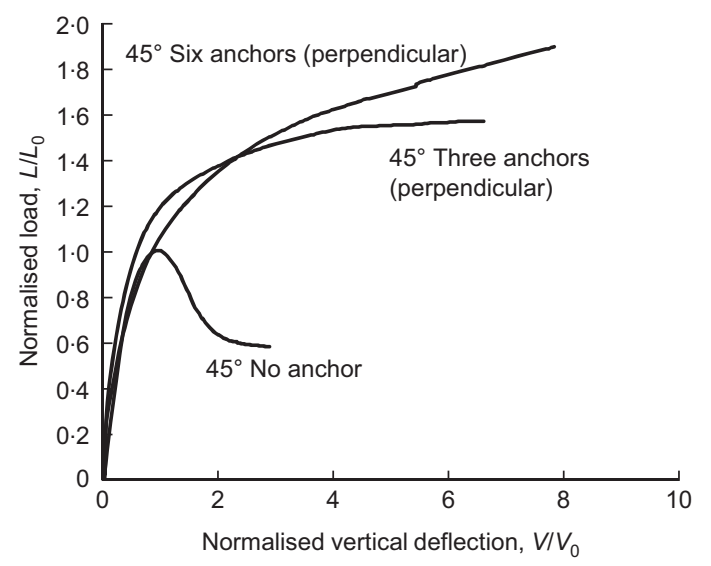

(a)

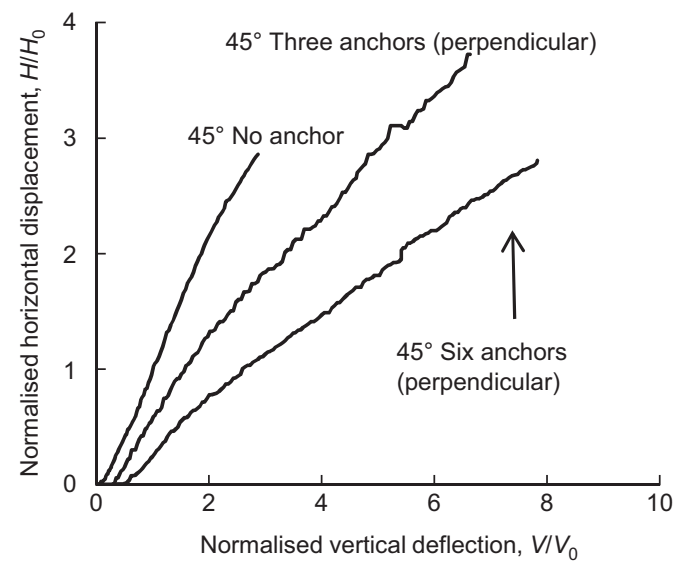

(b)

Fig. 4. Performance of slopes reinforced with three and six horizontal anchors ( $45^{\circ}$ slopes): (a) load versus vertical displacement; (b) horizontal and vertical displacement 


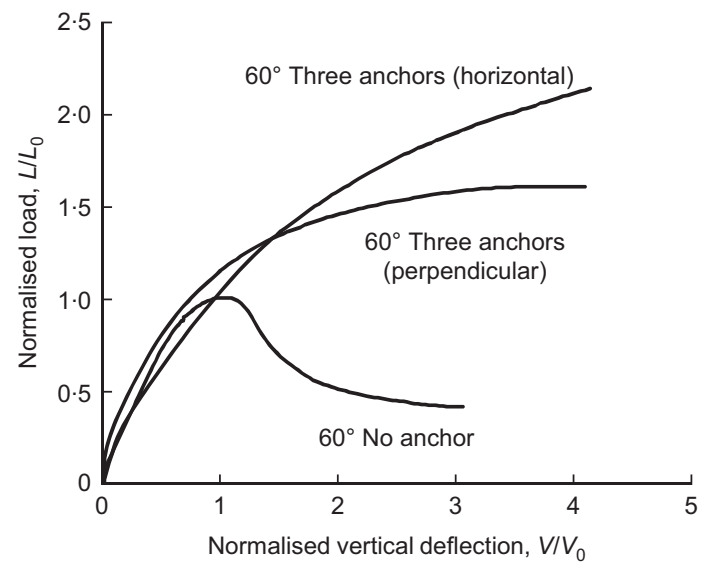

(a)

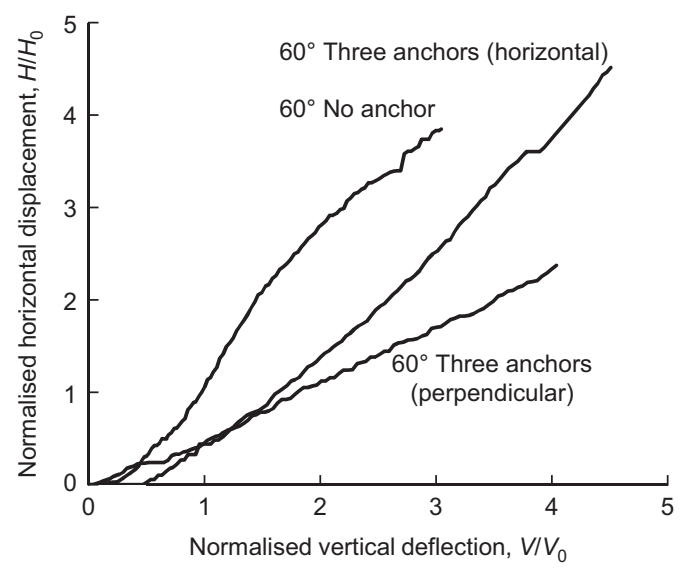

(b)

Fig. 5. Performance of slopes reinforced with three horizontal and perpendicular anchors $\left(60^{\circ}\right.$ slopes): (a) load versus vertical displacement; (b) horizontal and vertical displacement

and may have wider applications in practice, especially due to ease of installation.

In terms of application requirements, designers are concerned with both the depth of the anchor and the number of anchors needed. Sivakumar et al. (2013) showed that anchors operate in the same manner as granular columns. However, in the case of granular anchors, the bulging mechanism takes place at the bottom of the section. To ensure the effectiveness of the proposed system (bulging capacity), the tail of the anchor must be embedded to an absolute minimum of five times the anchor diameter behind the critical failure surface (Sivakumar et al., 2013). This is shown diagrammatically in Fig. 8 . The bulging capacity $P$ (or pull-out capacity) is given by (Hughes \& Withers, 1974)

$$
P=A\left(\frac{1+\sin \phi_{\mathrm{g}}^{\prime}}{1-\sin \phi_{\mathrm{g}}^{\prime}}\right)\left(\sigma_{\mathrm{vc}}+N_{\mathrm{c}}^{*} c_{\mathrm{u}}\right)
$$

in which $\sigma_{\mathrm{vc}}$ is the overburden pressure caused by the surrounding soil at the point of bulging, $\phi_{\mathrm{g}}^{\prime}$ is the angle of shearing resistance of the granular column, $c_{\mathrm{u}}$ is the undrained shear strength, $A$ is the cross-sectional area of the anchor and $N_{\mathrm{c}}^{*}$ is a bearing capacity factor that considers local shear failure. Therefore, the required anchor capacity can be calculated based on the depth of the anchors and the relevant slope strength parameters.

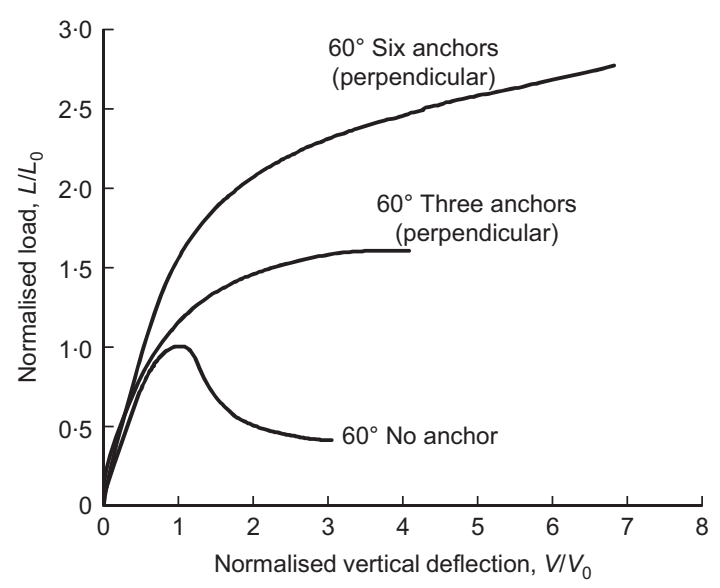

Fig. 6. Performance of slopes reinforced with three and six perpendicular anchors $\left(60^{\circ}\right.$ slope $)$
Using existing commercial software or analytical methods, the potential failure plane can be established, thereby allowing a designer to establish the minimum length of the granular anchors needed. Using the suggested equation, the pull-out capacity (or bulging capacity) of each anchor can be determined for the given strength parameters of the granular material and the surrounding soil. The analysis should then be iterated through until the designer achieves the required factor of safety for the slope configuration that will determine the number of anchors needed.

\section{CONCLUSION}

This research examined the influence of anchor numbers, orientation and side slope angle on the ultimate capacity of a cohesive slope. Granular anchors were proven to be an effective slope stabilisation technique for conventional and over-steepened cohesive slopes. Horizontal anchors performed better than perpendicular anchors (i.e. perpendicular to the slope). The observed enhanced performance in the case of horizontal anchors is attributed, to some extent, to the interaction between the steel tendon running through the granular column and the rigid loading plate. Significant strength and ductility increases can be easily achieved by the addition of anchors within the body of the mass to be retained. These were much more prominent in the case of

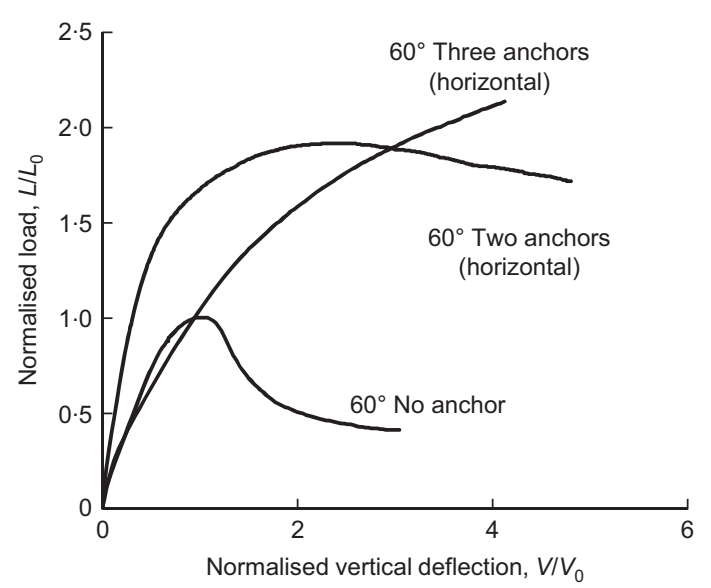

Fig. 7. Performance of slopes reinforced with two and three horizontal anchors ( $60^{\circ}$ slope) 


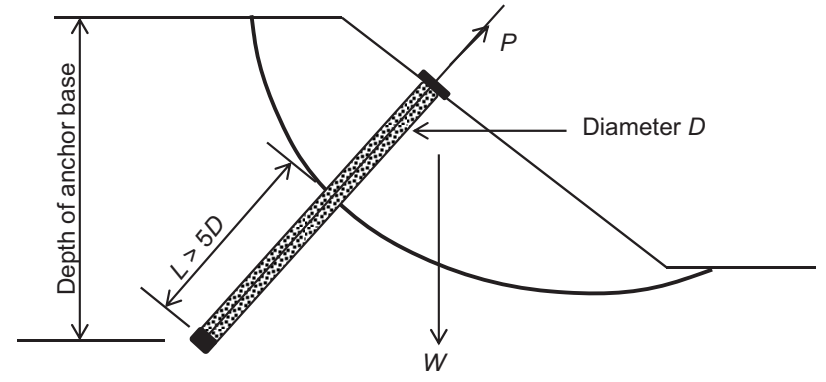

Fig. 8. Practical application

steep slopes $\left(60^{\circ}\right)$. Granular anchors also reduced the slope deformation significantly. Effective drainage is an additional benefit of stabilising slopes using granular anchors. Further research in the form of centrifuge modelling and full-scale study is planned in order to confirm the findings from the model study.

\section{ACKNOWLEDGEMENTS}

The authors would like to thank Mr Gordon Mackenzie, Geoman Ltd, Belfast for his constructive comments during the preparation of this article.

\section{REFERENCES}

Hughes, J. M. O. \& Withers, N. J. (1974). Reinforcing of soft cohesive soils with stone columns. Ground Engng 7, No. 3, 42-49.

Liu, K. F., Xie, X. Y., Zhang, J. F. \& Zhu, X. R. (2006). Compression/tension load capacity of stone column anchors. Proc. Inst. Civ. Eng. Geotech. Engng 159, No. 3, 161-165.

Madhav, M. R., Vidyaranya, B. \& Sivakumar, V. (2008). Analysis and comparison of displacement granular pile anchors. Proc. Inst. Civ. Eng. Ground Improve. 161, No. 1, 31-41.

Phanikumar, B. R. \& Ramachandra Rao, N. (2000). Increasing pull-out capacity of granular pile anchors in expansive soils using base geosynthetics. Can. Geotech. J. 37, No. 4, 870-881.

Phanikumar, B. R., Sharma, R. S., Srirama Rao, A. S. \& Madhav, M. R. (2004). Granular pile-anchor foundation (GPAF) system for improving the engineering behaviour of expansive clay beds. Geotech. Test. J. 27, No. 3, 279-287.

Phanikumar, B. R., Srirama Rao, A. \& Suresh, K. (2008). Field behaviour of granular pile-anchors in expansive soils. Proc. Inst. Civ. Eng. Ground Improve. 161, No. 4, 199-206.

Sivakumar, V., O'Kelly, B. C., Madhav, M. R., Moorhead, C. \& Rankin, B. (2013). Granular anchors under vertical loadingaxial pull. Can. Geotech. J. 50, No. 1, 123-132.

Srirama Rao, A., Phanikumar, B. R., Dayakar Babu, R. \& Suresh, K. (2007). Pullout behavior of granular pile-anchors in expansive clay beds in situ. ASCE J. Geotech. Geoenviron. Engng 133, No. 5, 531-538.

Whitlow, R. (1995). Basic soil mechanics, 3rd edn. Harlow: Longman Scientific and Technical.

\section{WHAT DO YOU THINK?}

To discuss this paper, please email up to 500 words to the editor at journals@ice.org.uk. Your contribution will be forwarded to the author(s) for a reply and, if considered appropriate by the editorial panel, will be published as a discussion. 